\title{
Desempeños Lingüístico, Cognitivo y Auditivo de un Grupo de Escolares con Trastorno Específico del Lenguaje
}

\author{
Linguistic, Cognitive and Auditory Performance \\ of a Group of School Children with Specific \\ Language Impairment
}

\section{RESUMEN}

Sandra Palma M. Fonoaudióloga Licenciada en Fonoaudiología Universidad de Chile

\section{Daniela Valdés I.}

Fonoaudióloga

Licenciada en Fonoaudiología Universidad de Chile

\section{Carmen Julia Coloma T. Académico Escuela de Fonoaudiología Universidad de Chile}

Contacto con los autores: Carmen Julia Coloma T. (Mg) Escuela de Fonoaudiología Universidad de Chile Independencia 1027 Santiago-Chile. Tel: (56 2) 9786181 Fax: (56 2) 9786608 e-mail: ccoloma@med.uchile.cl

Recibido: Noviembre, 2010 Aceptado: Mayo, 2011
El Trastorno Específico de Lenguaje (TEL) se define como una limitación significativa en la capacidad lingüística que no evidencia daño cerebral, desempeños cognitivos descendidos ni déficit motor. En nuestro medio, los escolares con TEL han sido poco estudiados. El propósito del trabajo es entregar información sobre aspectos lingüísticos, cognitivos y auditivos de escolares con TEL que cursan 10 básico. Se evaluó a 25 niños con TEL y 23 escolares sin TEL con las siguientes pruebas: Test para evaluar la Gramática Española de A. Toronto, Evaluación del discurso narrativo (EDNA), test de matrices progresivas coloreadas de Raven y un barrido audiométrico. Los resultados más importantes muestran que la mayoría de los niños con TEL presentan alguna alteración en las áreas evaluadas. En especial, se advierte que un gran porcentaje del grupo en estudio evidencia dificultades en los aspectos lingüísticos y en particular en el discurso narrativo. Por el contrario, un bajo porcentaje de ellos manifestó problemas en las habilidades cognitivas no verbales o un descenso en el umbral auditivo.

Palabras clave: Discurso Narrativo, Trastorno Específico de Lenguaje, Escolaridad.

\section{ABSTRACT}

Specific language impairment (SLI) is defined as a significant limitation in linguistic abilities that cannot be explained by brain damage, cognitive or motor disabilities. In our context, school children with SLI have not been fully investigated. The aim of this study is to obtain information about linguistic, cognitive and auditory aspects in 25 children with SLI attending year 1 of primary school and in 23 control children without SLI. The evaluation procedures included the Test para evaluar la Gramática Española de A. Toronto, the Evaluación del Discurso Narrativo (EDNA), the Raven's coloured progressive matrices and an audiometric screening. The results indicate that most of the children with SLI show deficits in the linguistic aspects assessed, specially in narrative speech. On the other hand, a few number of children with SLI presented with difficulties in non-verbal cognitive abilities and/or with hearing deficits.

Keywords: Specific language Impairment, narrative speech, school aged children.. 


\section{Introducción}

El Trastorno Específico del Lenguaje (TEL) es una limitación significativa en la capacidad lingüística de algunos niños que no evidencian pérdida auditiva, daño cerebral, desempeños cognitivos descendidos ni déficit motor. Además, se manifiesta en ausencia de restricciones socioambientales como son: la pobreza del input, las condiciones de vida extremas y la privación afectiva ${ }^{1}$. Por lo anterior, la determinación de este trastorno requiere de un diagnóstico basado en criterios de exclusión que permitan asegurar la ausencia de factores que expliquen el déficit de lenguaje.

Una de las alteraciones más frecuentes en los menores con TEL es la que se produce en el componente morfosintáctico. También, se postula que tal déficit es propio del TEL y por lo tanto, su existencia permite establecer la presencia de este cuadro. Al respecto, se sabe que los niños de habla inglesa con esta dificultad tienden a omitir los morfemas que indican pasado en los verbos regulares y los que marcan tercera persona singular ${ }^{2}$. En el caso de los menores hispanohablantes, las dificultades se advierten en el manejo de los artículos y en el uso de los pronombres clíticos ${ }^{3}$.

Además de la dificultad morfosintáctica, muchos de los sujetos con TEL evidencian problemas en el discurso narrativo. Así, se ha determinado que mantienen menos la coherencia, cometen errores de cohesión y presentan problemas con las categorías básicas de la narración ${ }^{4}$. La importancia de este tipo de discurso radica en que ha sido considerado como uno de los mejores predictores del éxito académico de los escolares con TEL. De este modo, los niños que narran adecuadamente en la etapa pre-escolar manifiestan posteriormente un buen desempeño académico por el contrario, aquellos con dificultades para relatar evidencian un rendimiento escolar descendido ${ }^{5}$. Es probable que lo anterior se deba a que la comprensión lectora se asocia con el discurso narrativo ${ }^{6}$.

Por otra parte, el problema lingüístico de los menores con TEL puede afectar su aprendizaje, en especial, el del lenguaje escrito. Por ello, existe gran interés en indagar sobre su lectura y escritura. En este ámbito, un foco central ha sido determinar la influencia de sus problemas lingüísticos en el desempeño lector que manifiestan en el periodo escolar ${ }^{7,8}$.

En Chile, los escolares con TEL asisten a escuelas con proyecto de integración. Para ser considerados en este tipo de establecimiento, el niño debe ser diagnosticado por el fonoaudiólogo y además haber sido evaluado integralmente para determinar las Necesidades Educativas Especiales derivadas del trastorno. Dicho diagnóstico se basa en las directrices entregadas en el "Decreto Supremo $170 " 9$ que lo define como un déficit significativo en el lenguaje oral que se manifiesta con un inicio tardío o un desarrollo lento y desviado del lenguaje. También alude a su especificidad al señalar que la dificultad no se explica por causas evidentes de tipo auditivo, neurológico, cognitivo o socioafectivo. En relación a la evaluación, se plantea considerar los niveles fonológico, léxico y morfosintáctico. Éste último incluye la comprensión y la expresión gramatical. Por otra parte, se especifica que las dislalias y los 
trastornos fonológicos por sí solos no son indicadores de TEL, por lo tanto, debe haber otro componente lingüístico comprometido. Finalmente, se propone completar el diagnóstico con la incorporación de alguna dificultad en la interacción comunicativa o en el desempeño académico del escolar.

A pesar de que las directrices para realizar el diagnóstico están definidas, en nuestro medio existe escasa información que permita caracterizar en diversos ámbitos a los alumnos con TEL. Ello es importante porque contar con una adecuada descripción posibilita comprender con mayor profundidad a estos niños en la etapa escolar. A su vez, dicha comprensión contribuye a tener más herramientas para apoyarlos pedagógica y fonoaudiológicamente en su aprendizaje del lenguaje escrito. Por lo anterior, el propósito del trabajo es entregar información sobre aspectos lingüísticos, cognitivos y auditivos de escolares con TEL que cursan $1^{\circ}$ básico.

\section{Método}

\section{Estudio}

La investigación es un estudio no experimental y descriptivo. Ello porque se evalúa la audición, las habilidades cognitivas no verbales, la gramática básica y el discurso narrativo en un momento específico sin intervenir ninguno de los aspectos mencionados. Además, es comparativo ya que se establecen las diferencias entre los desempeños del grupo en estudio y del grupo control.

\section{Sujetos}

El grupo en estudio estaba constituido por 25 escolares con diagnóstico de TEL asistentes a un colegio particular subvencionado con proyecto de integración. Todos los sujetos eran hombres y cursaban $1^{\circ}$ básico en el momento de la evaluación. Sus edades fluctuaban entre los 6 años 3 meses y los 7 años 10 meses. Su promedio fue de 6 años 11 meses.

El grupo control estuvo conformado por estudiantes sin problemas de lenguaje ni de aprendizaje, según lo informado por la fonoaudióloga y la profesora del nivel. Eran alumnos del mismo curso y establecimiento educacional de los estudiantes con TEL. Estaban pareados en edad (con un desfase de tres meses ya fuese sobre la edad o bajo la edad) y sexo con el grupo en estudio. De esta forma las edades del grupo control fluctuaron entre los 6 años 4 meses y 7 años 6 meses con un promedio de 6 años 11 meses.

\section{Instrumentos}

Se realizaron evaluaciones del ámbito cognitivo no verbal, auditivo y lingüístico. A continuación se presentan los instrumentos para medir los ámbitos no lingüísticos y posteriormente los correspondientes a los ámbitos lingüísticos.

Aspectos no lingüísticos

Evaluación audiométrica: Se utilizó audiómetro clínico marca Maico modelo MA41, calibrado según normas IEC 60645 -1, IEC 606452, ANSI S3.6, con auriculares TDH-39; otoscopio marca Riester modelo pen-scope ${ }^{\circledR}$. 
Evaluación de habilidades cognitivas no verbales: Se utilizó el Test de Matrices Coloreadas y Progresivas de Raven ${ }^{10}$. Su objetivo es medir habilidades cognitivas no verbales mediante la comparación de formas y del razonamiento por analogías visuales. Se puede aplicar a cualquier sujeto, independiente de su lengua, educación y capacidad verbal ${ }^{10}$. Consta de 36 ítems, los que están ordenados en dificultad progresiva y se responden sin la intervención del lenguaje.

Aspectos lingüísticos

Evaluación gramática básica: Se usó el Test Exploratorio de la Gramática Española de A. Toronto $(\mathrm{STSG})^{11}$ para medir gramática básica mediante la modalidad receptiva y expresiva. En relación a los análisis de confiabilidad, la subprueba expresiva obtiene un índice de 0.77 y la comprensiva logra un índice de 0.83 . Esta prueba está estandarizada en Chile para niños entre 3 años y 7 años 11 meses. Entrega criterios de corrección, puntajes promedio y percentiles de rendimiento.

Evaluación discurso narrativo: Se aplicó el procedimiento Evaluación del Discurso Narrativo $(E D N A)^{12}$. Su objetivo es medir el desempeño narrativo tanto a nivel expresivo como comprensivo. Cuenta con normas estandarizadas en Chile y percentiles y posee una escala de los desempeños de los niños que permite caracterizar sus narraciones cualitativamente.

\section{Procedimientos}

Se realizó una audiometría de barrido en las frecuencias 500, 1000, 2000 y $4000 \mathrm{~Hz}$, utilizando criterios internacionales ASHA (American Speech-Language-Hearing Association) que determinan normalidad auditiva a intensidades iguales o inferiores a $20 \mathrm{~dB}^{13}$. La audiometría se llevó a cabo en una sala silenciosa y sin distractores, donde estaba la examinadora y el niño. Se le explicó al menor lo que harían durante la evaluación con el fin de que se familiarice con el examen. Luego se efectuó una otoscopia para observar el estado del conducto auditivo externo y de la membrana timpánica del escolar. Posteriormente, se le entregaron las indicaciones para que identificara los tonos puros dados por los auriculares al momento de realizar el barrido. Se cuidó que comprendiera la instrucción antes de realizar el examen audiológico. Específicamente, la evaluación consistió en que el alumno respondió mediante la presión a un botón cada vez que percibía un tono. Para su interpretación, se consideró hipoacusia leve la pérdida que va desde los 20 hasta $40 \mathrm{~dB}$; moderada, la pérdida que se encuentra entre 40 y 60 dB. Además, se definió hipoacusia de conducción como aquella pérdida (desde leve a moderada) que se relaciona con el oído externo y medio. Por último, la hipoacusia severa fluctúa entre 60 y $80 \mathrm{~dB}$ y la profunda es aquella en que no se perciben sonidos o se logra escuchar sobre $80 \mathrm{~dB}^{13}$.

El test de Raven fue aplicado de manera individual en un ambiente protegido del ruido y otras distracciones. Se le explicó al niño que mirara un dibujo que consiste en un rectángulo coloreado que le falta una porción de distintas formas. Luego debía determinar, dentro de un set de 6 piezas, cuál era la parte que completaba el dibujo. La indicación 
específica es: "Mira con cuidado los dibujos (señalándolos), sólo una de las piezas completa bien el modelo (también se señala)", "muéstrame cuál es". La corrección consistió en asignar 1 punto a cada respuesta correcta y 0 a las incorrectas. Se utilizó la norma argentina ${ }^{10}$ para establecer el desempeño de los participantes. Según ella, los escolares con un rendimiento cognitivo no verbal adecuado fueron aquellos que obtuvieron puntajes que se ubicaban en o sobre el percentil 25 ( 6 años: 15 puntos o más, y 7 años: 22 puntos o más). Por su parte, los estudiantes que lograron puntuaciones correspondientes al percentil 10 o bajo este fueron considerados con déficit ( 6 años: 13 puntos o menos, y 7 años: 19 puntos o menos).

La evaluación del STSG fue individual y se efectuó en una sala con escaso ruido. Primero se aplicó la subprueba receptiva que mide 23 elementos sintácticos presentados en pares de oraciones. Además, las oraciones varían en una unidad sintáctica, de este modo si la categoría es el tiempo verbal, el ítem respectivo incluye dos oraciones opuestas. Por ejemplo, el ítem 8 expone dos oraciones que se oponen en el tiempo verbal: -El niño se vistió*, -El niño se viste. El asterisco indica que esa oración es la primera en ser solicitada. La respuesta se selecciona entre cuatro elementos y luego se señalan los estímulos verbales pedidos; estos estímulos se solicitan en un orden aleatorio especificado por la prueba.

\section{El instrumento expresivo posee 24} láminas, cada una de ellas ilustra una oración; la primera corresponde al ejemplo que clarifica la comprensión de la instrucción. En consecuencia, se evalúan 23 elementos. Su modalidad es la repetición de oraciones, por ello, el examinador dice dos oraciones referidas a dos imágenes distintas, luego señala una de las imágenes y pregunta: ¿cómo se llama esta? El niño repite lo que escuchó. Después se pregunta por la otra oración: ¿y esta?

La corrección de las dos subpruebas consiste en asignar 2 puntos cuando se responde a ambas oraciones, 1 punto si solo es una oración y 0 si se equivoca en las dos. Como se señaló, se miden 23 elementos sintácticos que implican dos oraciones que cuando están correctas se les asignan 2 puntos, por lo tanto, el puntaje máximo es 46 en cada subprueba. Para la interpretación de las puntuaciones se consideran los percentiles propuestos en la prueba. Así, los menores con problemas gramaticales son los que se ubican en el percentil igual o inferior a 10. Dicho desempeño en la subprueba receptiva corresponde a 35 puntos o menos para los niños de 6 y 7 años; en la subprueba expresiva el puntaje es equivalente o inferior a 25 a los 6 años y a los 7 años es igual o inferior a 35 puntos.

La aplicación de EDNA se realizó en una sala lo más tranquila posible para la realidad educativa. Se le dijo al alumno que la examinadora tenía un amigo enfermo que le gusta escuchar cuentos relatados por niños. Por eso le pedía que narrara unos cuentos que ella le iba a contar para que luego su amigo pudiera entretenerse con ellos. El sentido de la introducción a la tarea es justificar el relatar un cuento a una persona que ya lo conoce. Se utilizaron tres relatos ("la 
ardillita glotona", "el sapito saltarín" y "el lobo friolento"). Cada narración se lee con naturalidad y sin apoyo visual. El orden de la lectura es $1^{\circ}$ "la ardillita glotona", $2^{\circ}$ "el sapito saltarín" y $3^{\circ}$ "el lobo friolento". Luego de escuchar cada cuento, el niño lo relata y su narración se graba. Después, se le realizan preguntas del cuestionario establecido para el cuento; las respuestas en este caso, también son grabadas. De la misma forma se procede con los siguientes dos relatos.

La corrección de la producción de narraciones requirió primero analizarlas y posteriormente se les asignó el puntaje. El análisis se basó en criterios estructurales. Por ello, lo primero fue determinar si los cuentos están o no estructurados. Cuando los relatos infantiles carecieron de estructura se consignan los siguientes puntajes: no dice nada (0 punto), dice una o dos oraciones (0.25 puntos), aglutina acciones sin relación (0.5 puntos), agrupa oraciones en torno a un personaje ( 0.75 puntos) o produce secuencia de acción más obstáculo u obstáculo más resultado (0.75 puntos). Para los relatos infantiles con estructura se identificó cada categoría y elementos del cuento. A cada uno de ellos se les asigna un puntaje, es así como presentación completa tiene 1 punto, presentación incompleta 0.5 , episodio completo 2 puntos, incompleto 1 punto, el final abrupto, 1 punto, y el normal, 2 puntos.

Por su parte, en la comprensión se asignó 1 punto a las respuestas que son literales, 2 puntos a las que requieren inferencias y 0 a las incorrectas. El hecho de que a las respuestas inferenciales les corresponda una mayor puntuación es porque el sujeto debe efectuar relaciones entre la información explícita del texto y su conocimiento previo.

Se estableció que los escolares con dificultades en la producción o en la comprensión de discurso narrativo eran aquellos que se ubicaban en el percentil equivalente o inferior a 10. En producción este rendimiento corresponde a 9 puntos o menos, y en comprensión, a 19 puntos o a puntuaciones inferiores.

\section{Resultados}

El primer análisis consistió en determinar el porcentaje de escolares con dificultades en los ámbitos evaluados, tanto en el grupo en estudio como en el grupo control. Así, se advirtió que el $72 \%$ de los niños con TEL presentaron alteraciones y un 28\% de ellos evidenció un desempeño dentro de rangos típicos en todas las pruebas aplicadas. En el caso del grupo control, tiende a revertirse el fenómeno ya que un $70 \%$ manifestó rendimientos adecuados y solo un 30\% tuvo problemas en alguna de las mediciones realizadas.

Luego se estableció la distribución de las dificultades encontradas en ambos grupos, lo que se aprecia en el Gráfico 1. Es importante destacar que la mayoría de los sujetos con TEL evidenciaron alteraciones en más de uno de los ámbitos medidos. Por ejemplo, muchos escolares presentaron simultáneamente dificultades en gramática básica y en narración. Ello no se observó en los estudiantes del grupo control que solo evidenciaron problemas en una de las áreas lingüísticas (discurso narrativo o gramática básica). 


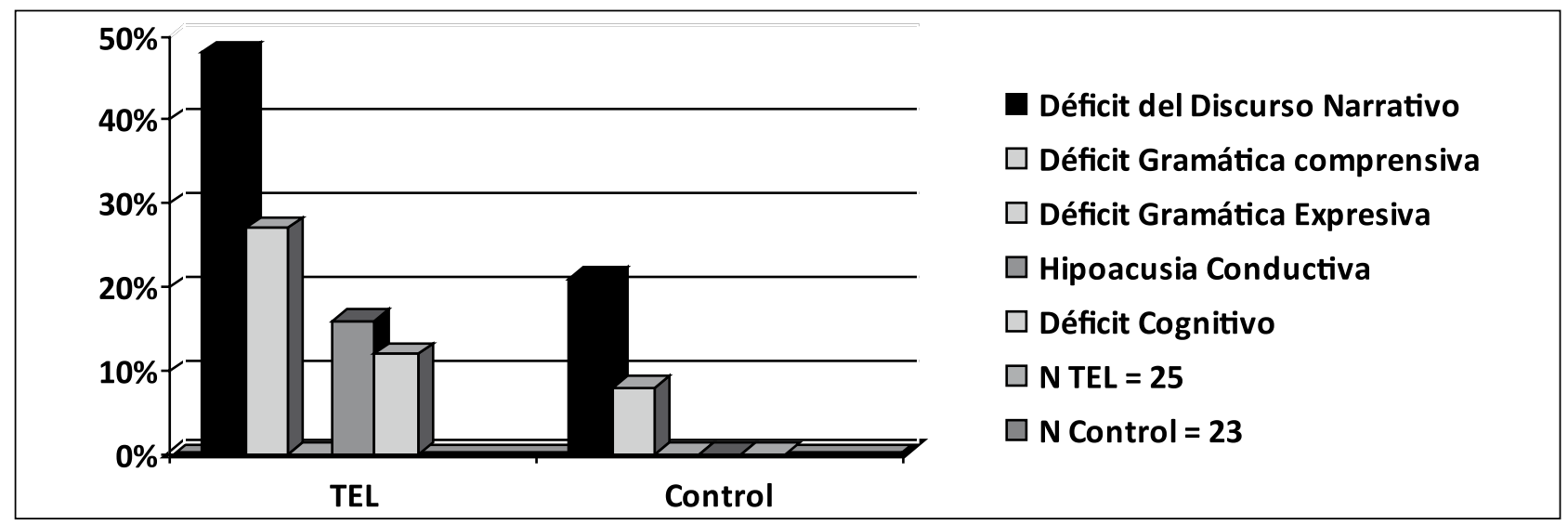

Gráfico 1. Distribución de las dificultades lingüísticas y no lingüísticas en niños con y sin trastorno específico de lenguaje.

Como se advierte en el gráfico 1, un porcentaje menor de escolares con TEL manifestó hipoacusia de conducción de carácter leve o déficit cognitivo no verbal. También sucedió que hubo un niño con TEL que tuvo hipoacusia leve $\mathrm{y}$ déficit cognitivo.

Con respecto a la gramática básica, tanto los escolares del grupo en estudio como los del grupo control manifestaron dificultades sólo en comprensión. Al respecto, es necesario indicar que la proporción de los menores con TEL que evidencian dichas dificultades es tres veces mayor que la del grupo control.

Por otra parte, la presencia de dificultades en el discurso narrativo concentró el mayor porcentaje de participantes de ambos grupos (48\% de los sujetos con TEL y $21 \%$ menores del grupo control). No obstante, se debe señalar que el porcentaje de niños diagnosticados con TEL con déficit narrativo es el doble que el del grupo control.

Por lo anterior, se analizó especialmente este plano en relación al compromiso de la producción y comprensión de narraciones. Se consideró para este análisis a la totalidad de los niños de cada grupo que presentó dificultad en algún ámbito narrativo, lo que significó trabajar con 12 alumnos con TEL y 8 sin problemas lingüísticos. El gráfico 2 ilustra la distribución de los escolares considerando este criterio.

Como se advierte en el gráfico 2, un grupo de escolares con TEL evidencia dificultades tanto en la comprensión como en la producción de narraciones. En cambio, el grupo control solo manifiesta alteraciones en la elaboración de cuentos y su comprensión de narraciones es adecuada. Los datos comentados indican que ambos grupos muestran compromiso en la producción de narraciones.

Debido a que la dificultad en producción de relatos fue la más frecuente y común en ambos grupos, pareció interesante estudiar con mayor profundidad este aspecto. Así, basándose en la escala de desarrollo narrativo ${ }^{12}$ se distinguieron dos niveles diferentes de compromiso: leve y severo. El compromiso leve implica que el escolar relata con presentación y episodio pero 


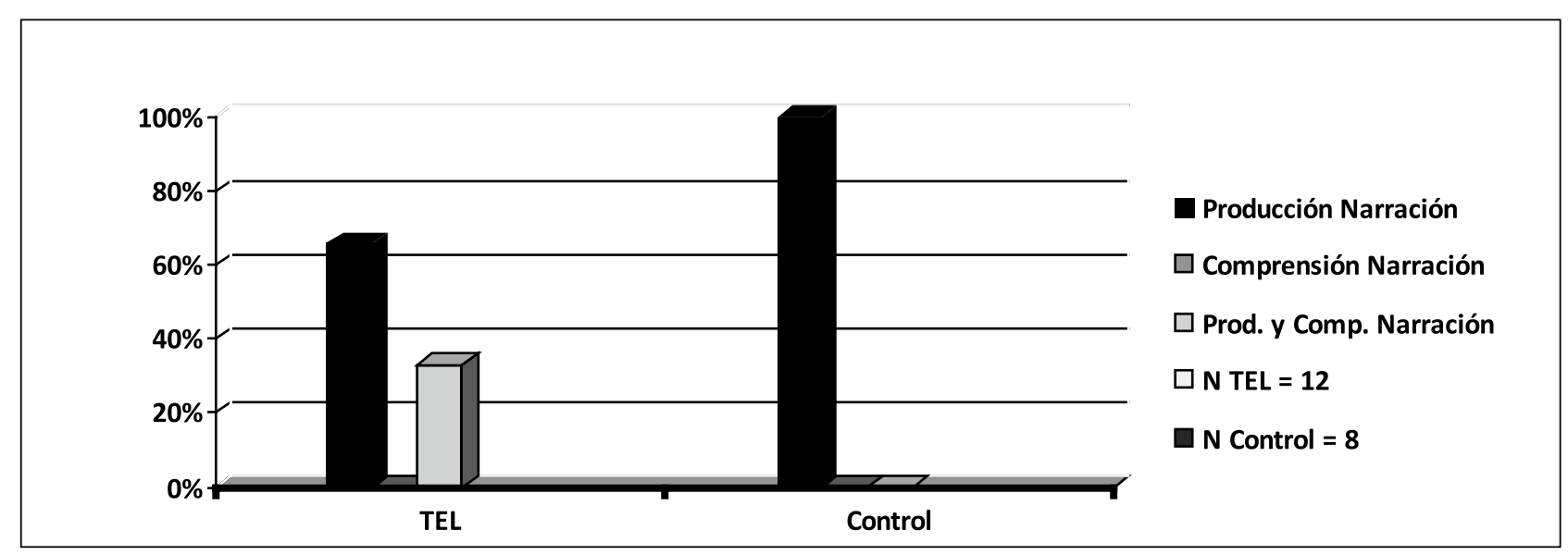

Gráfico 2. Distribución del compromiso en la producción y comprensión de narraciones en niños con y sin trastorno específico de lenguaje.

sin completar una de estas categorías, mientras que el severo se manifiesta cuando el niño no logra estructurar su discurso narrativo. Esta distinción se sustenta en que entre los 6 y 7 años los menores estructuran sus relatos con presentación y episodio, ambos completos ${ }^{12}$. Se observó que un $75 \%$ de los escolares con TEL manifestó dificultades severas en la producción narrativa y un $25 \%$ presentó alteraciones de tipo leve. Por su parte, la totalidad de los niños del grupo control manifestaron un déficit narrativo severo.

Como ya se comentó, una de las características del desempeño del grupo en estudio es que tienen alteraciones en más de una de las áreas evaluadas, lo que no sucede con los niños sin problemas de lenguaje. Es así como el 33\% de los sujetos que muestran dificultades discursivas severas también evidencian alteraciones en la comprensión de la gramática básica. En cambio, si se considera a los sujetos con compromiso morfosintáctico, el $42 \%$ de ellos tiene dificultades en la gramática básica junto a un severo déficit en el discurso narrativo.
En síntesis, los aspectos lingüísticos son los que aparecen comprometidos en un mayor porcentaje de escolares con TEL, en especial, la narración. Ello es relevante porque puede afectar su escolaridad ya que el discurso narrativo se asocia con la comprensión lectora.

\section{Discusión}

El propósito del trabajo fue entregar información sobre aspectos lingüísticos, cognitivos no verbales y auditivos de escolares con TEL que cursan $1^{\circ}$ básico. En tal sentido interesó describir dichos aspectos para contribuir a caracterizarlos y no cuestionar el diagnóstico de trastorno específico del lenguaje de la muestra.

Considerando esta perspectiva, la investigación mostró que un grupo importante de escolares con TEL presentaban problemas en algunas de las áreas evaluadas (audición, cognición no verbal, gramática básica o narración), fenómeno que no sucedió en los participantes sin problemas de lenguaje. 
Así, en el grupo control existieron escolares con rendimientos disminuidos en comprensión de gramática básica (8.6\%) o déficit en producción de narraciones (21\%). Lo anterior sugiere que manifiestan algún grado de dificultad lingüística que debe ser atendida. El que los menores con problemas en dichos ámbitos pasaran desapercibidos puede deberse a que utilizan estrategias funcionales que les permiten comunicarse adecuadamente con sus pares y profesores. Además, el contar con escasa información sobre el desempeño lingüístico esperado a los 6 años también contribuye a que sea difícil la identificación de sujetos con problemas de lenguaje en este periodo.

Los datos también instan a reflexionar sobre las estrategias de despistaje que se utilizan en los establecimientos para determinar el rendimiento lingüístico de un estudiante. Al parecer se requiere de herramientas más específicas que permitan establecer los déficit de lenguaje más característicos del periodo escolar.

Otro hallazgo fue que existieron participantes con TEL que no manifestaron rendimientos disminuidos en ninguna de las pruebas aplicadas. Es posible que lo anterior se deba a que las mediciones realizadas no incluyeron aspectos que podrían estar deficitarios como es la sintaxis compleja que es característica del desarrollo del lenguaje en la etapa escolar ${ }^{14}$. Por ello, es necesario que en futuros estudios se aborden dimensiones lingüísticas que se adquieren en este periodo.
Como se mencionó, los niños con TEL fueron los únicos que presentaron problemas en audición y cognición no verbal aunque el número que mostró este tipo de dificultades fue escaso. Por lo tanto, no parece ser característico de este grupo manifestar rendimientos descendidos en tales ámbitos. Ello no es sorprendente porque el TEL se define como una dificultad significativa en la capacidad lingüística ${ }^{1}$ y su diagnóstico se realiza descartando déficits en áreas no lingüísticas tales como la audición y la cognición no verbal.

Los sujetos con problemas en audición presentaron hipoacusia de conducción leve que puede ser provocada por una otitis media y por ello en estos casos es transitoria. Este tipo de hipoacusia se caracteriza por no ser evidente, en consecuencia, su identificación requiere de una evaluación audiométrica. Por otra parte, el carácter leve de dicha dificultad no permite explicar el problema lingüístico. No obstante, es posible que los escolares con TEL que presentan hipoacusia leve evidencien un descenso en la discriminación auditiva de fonemas, lo que compromete el aprendizaje de la lectura debido a que la capacidad de asociar fonemas a grafemas puede alterarse. Lo anterior significa que en la intervención deben existir estrategias específicas que consideren un posible problema auditivo en algunos estudiantes con TEL.

El déficit en las habilidades cognitivas no verbales que se encontró en un porcentaje de escolares con TEL muestra que tienen limitaciones en la capacidad para establecer relaciones analógicas visuales. Este tipo de 
habilidades se relaciona con la atención, la función ejecutiva prefrontal y con diferentes tipos de memoria (visual, procedural y de trabajo). Los ámbitos cognitivos mencionados son relevantes para un buen desempeño escolar, lo que posibilita hipotetizar que estos menores podrían evidenciar dificultades en algún área de su aprendizaje. Por lo tanto, requieren de una intervención específica, además de la lingüística, que les permita enfrentar las exigencias escolares.

También, habría que considerar una evaluación psicométrica para obtener mayor información sobre su desempeño cognitivo general y así establecer prioridades en el apoyo que se brindará.

Por otra parte, fue característico del grupo en estudio la coexistencia de los problemas en discurso narrativo y en comprensión de gramática básica. Dicha coexistencia sugiere que el núcleo problemático es lingüístico, lo que es esperable ya que la dificultad de lenguaje en este cuadro es lo determinante ${ }^{15}$. Se debe señalar que dicha coexistencia se dio en aquellos niños con problemas severos del discurso, es decir, correspondió a los escolares que no estructuraban sus relatos. Desde esta perspectiva es posible pensar que una de las variables que podrían afectar la adquisición de la estructura discursiva es la comprensión morfosintáctica simple.

Comoya se planteó, los resultados obtenidos indican que en ambos grupos el compromiso en los aspectos lingüísticos es mayor, en especial las dificultades narrativas. Al respecto, se advirtió que los problemas en producción de narraciones se produjeron tanto en los escolares del grupo en estudio como en los estudiantes del grupo control. Sin embargo, en los sujetos con TEL se observó que en algunos casos esta dificultad se daba junto a problemas en comprensión de relatos.

Una explicación a la menor cantidad de escolares con TEL que evidencian problemas en comprensión puede deberse a que la prueba que mide este aspecto está constituida por ítems que en su mayoría son fáciles para niños de 6 y 7 años $^{12}$. Por ello, es posible que no permita detectar con precisión los problemas que puedan manifestar estos sujetos. En consecuencia, se requiere profundizar con otro tipo de mediciones los posibles problemas en comprensión de relatos.

En relación a la producción de narraciones, se determinó que un grupo importante de sujetos con TEL presentaban problemas severos en la elaboración de narraciones, lo que se evidenciaba en que sus relatos carecían de estructura. Lo anterior revela que es una tarea lingüística exigente $y$, por lo tanto, es importante abordarla de forma específica. En la actualidad la evaluación de este tipo de discurso no está especificada en el "Decreto Supremo 170", por consiguiente, la planificación de un tratamiento específico para el déficit en narraciones generalmente no es considerada.

Lo expuesto es preocupante ya que existe un grupo de niños con TEL que no desarrolla su discurso narrativo con la experiencia escolar y tratamiento fonoaudiológico centrado en las otras 
habilidades lingüísticas ${ }^{16}$. Debido a su importancia para la comprensión lectora, se requiere que parte de la intervención se centre en incrementar este tipo de discurso. También es necesario desarrollar planes sistemáticos de estimulación de narraciones en el aula para todos los escolares. Ello porque existe la posibilidad de que alumnos sin TEL tengan dificultades narrativas.

Finalmente, esta investigación consideró solo algunos aspectos de los escolares con TEL, lo que permitió mostrar que el discurso narrativo es relevante y por eso debe ser considerado en el abordaje de estos escolares. No obstante, es necesario estudiar otros aspectos tanto lingüísticos como no lingüísticos con el fin de lograr una caracterización más completa que permita apoyarlos más efectivamente. Al respecto, futuros trabajos deberían recabar información sobre el rendimiento académico, el desempeño lector, la sintaxis compleja, el léxico, entre otros.

\section{Referencias}

1. Aguado, G. (1999) Trastorno específico del lenguaje. Retraso del lenguaje y disfasia. Archidona (Málaga): Aljibe.

2. Rice, M.L.; Wexler, K. \& Redmond, S.M. (1999) Grammaticality judgments of an extended optional infinitive grammar: Evidence from English-speaking children with specific language impairment. J Speech Lang Hear Res, 42, 943-961.

3. Simon-Cereijido, G. \& Gutiérrez-Clellen, V.F. (2007) Spontaneous language markers of Spanish language impairment. App Psycho, 28, 317-339.

4. Buiza, J.J.; Adrián-González, M. \& RodríguezParra, M.J. (2004) Evaluación de los marcadores psicolingüísticos en el diagnóstico del trastorno específico del lenguaje. Rev Logop Fon Audiol, 24(4): 142155.
5. Contreras, M.C. \& Soriano, M. (2004) El valor de la narrativa en la caracterización de los alumnos con dificultades del Lenguaje, Rev Logop Fon Audiol, 24, 119125.

6. Naremore R.; Densmore, A. \& Harman, D. (2001) Assessment and treatment of school age language disorders: a resource manual. Canadá: Singular. Thomson Learning.

7. Catts, H. W.; Fey, M.E.; Tomblin, B. \& Zhang, X. (2002) A longitudinal investigation of reading outcomes in children with language impairment. J Speech Lang Hear Res, 45(6): 1142-1157.

8. Bishop, D.V.M. \& Snowling, M.J. (2004) Developmental dyslexia and specific language impairment: same or different? Psychol Bull, 130, 858-888.

9. Decreto Supremo No. 170 del Ministerio de Educación del Gobierno de Chile (2009). Recuperado de http://www.mineduc.cl/biblio/ documento/201005031126500.DEC200900170.pdf

10. Raven, J. C. (2005) Test de matrices progresivas. Escalada Coloreada. Buenos Aires: Paidós.

11. Pavez, M.M. (2010) Test Exploratorio de la Gramática Española de A. Toronto, aplicación en Chile. Santiago, Chile: Ediciones Universidad Católica de Chile.

12. Pavez, M.M.; Coloma, C. \& Maggiolo, M. (2008) El Desarrollo Narrativo en Niños. Barcelona, España: Ars Médica.

13. Asha Web Site, Mayo (2010) Types, Degree and configuration of hearing loss. Audiology information series http: //search.asha.org/default. aspx?q=degree $\% 20$ hearing $\% 20$ loss

14. Nippold, M. (1998) Later language development: the school age and adolescent years. Texas: Pro-Ed.

15. Fresneda, D. \& Mendoza, E. (2005) Trastorno Específico del lenguaje: concepto, clasificaciones y criterios de identificación. Rev Neurol, 41, 51-56.

16. Coloma, C.J. \& Alarcón, P. (2009) El discurso narrativo y la comprensión lectora en escolares con trastorno específico de lenguaje. Revista de Psicología General y Aplicada, 62 (3): 147-156. 